\title{
EDMs of the nucleon and light nuclei in Chiral Effective Theory
}

\section{Emanuele Mereghetti*}

$L B N L$

E-mail: emereghetti@lbl.gov

I present the calculation of the EDM of the nucleon, deuteron, helion and triton induced by the QCD $\bar{\theta}$ term, and by the minimal set of dimension-six parity and time-reversal violating operators. Using the tools of chiral EFT, I discuss how different symmetry properties of $P$ and $T$ violating operators at the quark-gluon level imply qualitatively different relations between observables in the one, two and three-nucleon sector. If experimentally observed, these relations would provide important clues to connect time-reversal violation in hadronic systems to its fundamental, microscopic source.

Xth Quark Confinement and the Hadron Spectrum

8 - 12 October 2012

TUM Campus Garching, Munich, Germany

\footnotetext{
* Speaker.
} 


\section{Introduction}

Permanent electric dipole moments (EDMs) of particles, nuclei, atoms, and molecules are powerful probes of physics beyond the Standard Model (SM) [1]. EDMs are signal of parity $(P)$ and time reversal $(T)$ violation $(P F)$ in the flavor diagonal sector. Therefore, they are insensitive, within the current experimental sensitivity, to the only measured $C P$-violating parameter in the $\mathrm{SM}$, the phase of the Cabibbo-Kobayashi-Maskawa (CKM) matrix. The SM has another source of PPT, the QCD $\bar{\theta}$ term. The $\bar{\theta}$ angle is a free parameter in the QCD Lagrangian, and thus, $a$ priori, a $\mathscr{O}(1)$ number. However, the stringent bound on the neutron EDM, $\left|d_{n}\right|<2.9 \cdot 10^{-13} e$ fm [2], constrains $\bar{\theta}$ to be unnaturally small, $\bar{\theta} \lesssim 10^{-10}$. Because of the smallness of $\bar{\theta}$, and the negligible contribution from the CKM, $C P$ violation from physics beyond the SM could provide an important, even leading, contribution to EDMs. As a consequence, a rich experimental program in under way, with the goal to improve the bounds on the neutron and atomic EDMs by one/two orders of magnitude by the end of the decade. Furthermore, new techniques have been proposed to measure the EDMs of light ions (the proton, the deuteron, and, possibly, other light nuclei) directly, and with the same accuracy as EDMs of neutral particles, in storage ring experiments [3].

A positive signal in any of the next-generation experiments would be a clear signal of $\not P T$ beyond the CKM phase. But, will we be able to identify the dominant, microscopic mechanism(s) that generates it? Is the $\bar{\theta}$ term, or physics at some high energy scale? In this talk, I address some aspects of this multifaceted question. I focus on hadronic EDMs, and I discuss, using the tools of nuclear Effective Field Theories (EFTs), how different symmetry properties of $\not P T$ operators at the quark-gluon level imply qualitatively different relations between observables in the one, two and three-nucleon sector. The observation of these relations in EDM experiments, then, would provide strong hints on the nature of the dominant $\not P T$ source.

\section{2. $\not P T$ interactions at the quark-gluon and hadronic level}

The first $\not P T$ source I consider is the QCD $\bar{\theta}$ term. By performing a $U_{A}(1)$ rotation, the $\bar{\theta}$ term can be written as a complex mass term, which, after imposing vacuum alignment [ $₫$, is

$$
\mathscr{L}_{4}=-m_{\star} r^{-1}(\bar{\theta}) \sin \bar{\theta} \bar{q} i \gamma^{5} q
$$

where $q$ is a doublet of the two lightest quarks $q=(u, d), m_{\star}$ is the light quark reduced mass, $m_{\star}=m_{u} m_{d} /\left(m_{u}+m_{d}\right)$ and the function $r(\bar{\theta})$ is approximately 1 for small $\bar{\theta}$.

The smallness of $\bar{\theta}$ can make $\not P T$ beyond the SM competitive, if not dominant. We assume new physics to be characterized by some high energy scale $M_{\mathcal{F}}$, much larger than the electroweak (EW) scale. At the EW scale, new physics is integrated out, giving rise to gauge invariant operators of higher dimension. The most important are, presumably, the operators of the lowest dimension, that is dimension-six operators. For nuclear observables, the $\not P T$ Lagrangian at the EW scale must be run down to the typical hadronic scale, $\mu \sim 1 \mathrm{GeV}$, and, in the process, heavy SM particles are integrated out. After doing so, the minimal set of dimension-six operators includes the quark electric and chromo-electric dipole moments (qEDM and qCEDM), the gluon chromo-electric dipole 
moment (gCEDM), and four four-quark operators [5, 6].

$$
\begin{aligned}
\mathscr{L}_{6}= & -\frac{1}{2} \bar{q}\left(d_{0}+d_{3} \tau_{3}\right) i \sigma^{\mu v} \gamma_{5} q F_{\mu v}-\frac{1}{2} \bar{q}\left(\tilde{d}_{0}+\tilde{d}_{3} \tau_{3}\right) i \sigma^{\mu v} \gamma_{5} \lambda^{a} q G_{\mu \nu}^{a} \\
& +\frac{d_{W}}{6} f^{a b c} \varepsilon^{\mu v \alpha \beta} G_{\alpha \beta}^{a} G_{\mu \rho}^{b} G_{v}^{c \rho} \\
& +\frac{\operatorname{Im} \Xi_{1}}{4} \varepsilon^{3 i j} \bar{q} \tau^{i} \gamma^{\mu} q \bar{q} \tau^{j} \gamma_{\mu} \gamma_{5} q+\frac{\operatorname{Im} \Xi_{8}}{4} \varepsilon^{3 i j} \bar{q} \tau^{i} \gamma^{\mu} \lambda^{a} q \bar{q} \tau^{j} \gamma_{\mu} \lambda^{a} \gamma_{5} q \\
& +\frac{\operatorname{Im} \Sigma_{1}}{4}\left(\bar{q} q \bar{q} i \gamma_{5} q-\bar{q} \vec{\tau} q \cdot \bar{q} \vec{\tau} i \gamma_{5} q\right)+\frac{\operatorname{Im} \Sigma_{8}}{4}\left(\bar{q} \lambda^{a} q \bar{q} i \gamma_{5} \lambda^{a} q-\bar{q} \vec{\tau} \lambda^{a} q \cdot \bar{q} \vec{\tau} i \gamma_{5} \lambda^{a} q\right) .
\end{aligned}
$$

$d_{0}\left(\tilde{d}_{0}\right)$ and $d_{3}\left(\tilde{d}_{3}\right)$ represent the isoscalar and isovector components of the qEDM (qCEDM). These are dimension-five operators, but, since they break $S U_{L}(2)$, they must be proportional to the Higgs vacuum expectation value, making them of effective dimension six. $d_{W}$ is the gCEDM. Of the four-quark operators, $\Sigma_{1,8}$ are invariant under the SM gauge group, and can be generated directly at the EW scale. $\Xi_{1,8}$ are not invariant under $S U_{L}(2)$, and are generated by integrating out the weak bosons [ד]. I define the couplings in Eq. (2.2) as

$$
\begin{aligned}
& d_{0,3}=\mathscr{O}\left(\frac{e \delta \bar{m}}{M_{\mp}^{2}}\right), \quad \tilde{d}_{0,3}=\mathscr{O}\left(4 \pi \frac{\tilde{\delta} \bar{m}}{M_{\mp}^{2}}\right), \quad d_{W}=\mathscr{O}\left(4 \pi \frac{w}{M_{T}^{2}}\right), \\
& \Xi_{1,8}=\mathscr{O}\left(\frac{(4 \pi)^{2} \xi}{M_{\mp}^{2}}\right), \quad \Sigma_{1,8}=\mathscr{O}\left(\frac{(4 \pi)^{2} \sigma}{M_{T}^{2}}\right),
\end{aligned}
$$

in terms of dimensionless numbers $\delta, \tilde{\delta}, w, \xi$, and $\sigma$. The size of these parameters depends on the exact mechanisms of electroweak and $P$ and $T$ breaking, and on the running to low energies.

The next step is to calculate hadronic observables from the Lagrangian in Eqs. (2.1) and (2.2). To do so requires a complete solution of QCD, which is not in sight. There exist lattice calculations of the nucleon EDM for the $\bar{\theta}$ term [8, 9], but they are not at the physical pion mass yet, and an extension to light nuclei is non trivial. On the other hand, the chiral symmetry of QCD and its spontaneous breaking allow to develop an EFT, chiral perturbation theory, to describe the interactions of pions, nucleons and photons at energies $Q$ much smaller than the strong interaction scale, $M_{\mathrm{QCD}} \sim 1$ $\mathrm{GeV}$. The PPT EFT Lagrangian stemming from Eqs. (2.1) and (2.2) can be obtained by constructing all the operators that transform under $S U_{L}(2) \times S U_{R}(2)$ as the sources at the quark-gluon level, and by assigning them a scaling in the EFT expansion parameter, $Q / M_{\mathrm{QCD}}$. Unfortunately, the couplings in the EFT cannot be derived from QCD, but they can be estimated by naive dimensional analysis (NDA), and extracted from data, when enough observables are available.

It is therefore important to look at the transformation properties of the PPT operators in Eqs. (2.1) and (2.2). The $\bar{\theta}$ term and qCEDM break chiral symmetry as components of $S O(4)$ vectors. The $\bar{\theta}$ term and isoscalar qCEDM respect isospin, while the isovector component of the qCEDM breaks also isospin. The qEDM has the same transformation properties as the qCEDM, with the important difference that if one is interested in purely hadronic operators, e.g $\not P T$ pion-nucleon and nucleon-nucleon couplings, the photon needs to be integrated out, causing a large suppression of $\alpha_{\mathrm{em}} / 4 \pi$. The gCEDM and the two four-quark operators $\Sigma_{1,8}$ are chiral invariant. Having the same transformation properties, they generate exactly the same couplings in the EFT, so that in our approach their effects are not distinguishable. I will discuss them together, and denote them 
collectively as chiral invariant sources ( $\chi \mathrm{ISs}$ ). $\Xi_{1,8}$, which, because of their properties I will call four-quark left-right operators (FQLR), break chiral symmetry and isospin, as the 34 component of a symmetric tensor.

The EFT Lagrangian induced by the PPT operators up to dimension-six was discussed in detail in Refs. [10, 6]. For the observables I discuss here, the most important couplings are

$$
\begin{aligned}
\mathscr{L}_{P \| t}= & -\frac{\bar{g}_{0}}{F_{\pi}} \vec{\pi} \cdot \bar{N} \vec{\tau} N-\frac{\bar{g}_{1}}{F_{\pi}} \pi_{3} \bar{N} N+\bar{C}_{1} \bar{N} N \partial_{\mu}\left(\bar{N} S^{\mu} N\right)+\bar{C}_{2} \bar{N} \vec{\tau} N \partial_{\mu}\left(\bar{N} S^{\mu} \vec{\tau} N\right) \\
& -2 \bar{N}\left(\bar{d}_{0}+\bar{d}_{1} \tau_{3}\right) S^{\mu} v^{v} N F_{\mu \nu},
\end{aligned}
$$

where $F_{\pi}=186 \mathrm{MeV}$. The relative importance of the couplings in Eq. (2.4) depends on the transformation properties of the $P P T$ source at the quark-gluon level. For sources that break chiral symmetry, the non-derivative pion-nucleon couplings appear at lowest order in the PPT EFT Lagrangian, with nucleon-nucleon and nucleon-photon couplings suppressed by two powers of $Q / M_{\mathrm{QCD}}$. The main difference between the $\bar{\theta}$ term and the qCEDM is that the qCEDM, having both an isoscalar and isovector component, generates $\bar{g}_{0}$ and $\bar{g}_{1}$ at the same level, while for the $\bar{\theta}$ term $\bar{g}_{1}$ is suppressed by $\varepsilon m_{\pi}^{2} / M_{\mathrm{QCD}}^{2}$ with respect to $\bar{g}_{0}$. The FQLR is purely isovector, but does contribute to $\bar{g}_{0}$ in LO, because of vacuum alignment [6]. In the case of the qEDM, the purely hadronic operators $\bar{g}_{0,1}$ and $\bar{C}_{1,2}$ are suppressed by $\alpha_{\mathrm{em}} / 4 \pi$, and they are irrelevant for all the observables we consider. The most important couplings are the short-range contributions to the isoscalar and isovector nucleon EDMs $\bar{d}_{0}$ and $\bar{d}_{1}$. For $\chi \mathrm{ISs}$, in order to generate the chiral breaking couplings $\bar{g}_{0}$ and $\bar{g}_{1}$, an insertion of the quark mass is needed, which costs two powers of $Q / M_{\mathrm{QCD}}$. On the other hand, $\bar{C}_{1,2}$ and $\bar{d}_{0,1}$ are not suppressed, so that all the interactions in Eq. (2.4) are of the same order.

In summary, the pion-nucleon coupling $\bar{g}_{0}$ and $\bar{g}_{1}$ scale as

$$
\begin{aligned}
& \bar{g}_{0}=\mathscr{O}\left(\bar{\theta} \frac{m_{\pi}^{2}}{M_{\mathrm{QCD}}}, \tilde{\delta} \frac{m_{\pi}^{2} M_{\mathrm{QCD}}}{M_{\mathcal{T}}^{2}}, w \frac{m_{\pi}^{2} M_{\mathrm{QCD}}}{M_{\mathcal{T}}^{2}}, \xi \frac{\varepsilon M_{\mathrm{QCD}}^{3}}{M_{\mathcal{T}}^{2}}\right), \\
& \bar{g}_{1}=\mathscr{O}\left(\bar{\theta} \frac{\varepsilon m_{\pi}^{4}}{M_{\mathrm{QCD}}^{3}}, \tilde{\delta} \frac{m_{\pi}^{2} M_{\mathrm{QCD}}}{M_{\mathcal{T}}^{2}}, w \varepsilon \frac{m_{\pi}^{2} M_{\mathrm{QCD}}}{M_{\mathcal{T}}^{2}}, \xi \frac{M_{\mathrm{QCD}}^{3}}{M_{\mathcal{T}}^{2}}\right) .
\end{aligned}
$$

The short-range contributions to the isosclar and isovector nucleon EDM scale as

$$
\bar{d}_{0,1}=\mathscr{O}\left(\bar{\theta} \frac{m_{\pi}^{2}}{M_{\mathrm{QCD}}^{3}}, \tilde{\delta} \frac{m_{\pi}^{2}}{M_{\mathrm{QCD}} M_{\mp}^{2}}, \delta \frac{m_{\pi}^{2}}{M_{\mathrm{QCD}} M_{\mp}^{2}}, w \frac{M_{\mathrm{QCD}}}{M_{\mp}^{2}}, \xi \frac{M_{\mathrm{QCD}}}{M_{\mp}^{2}}\right) .
$$

The four-nucleon couplings $\bar{C}_{1,2}$ are only relevant for the $\chi \mathrm{ISs}$, for which they scale as $\bar{C}_{1,2}=$ $\mathscr{O}\left(w M_{\mathrm{QCD}} / F_{\pi}^{2} M_{\mathcal{f}}^{2}\right)$, the same order as $\bar{g}_{0,1}$ and $\bar{d}_{0,1}$. However, $\bar{C}_{1,2}$ are only relevant to the ${ }^{3} \mathrm{He}$ and ${ }^{3} \mathrm{H}$ EDMs, and, though expected to be leading, their contribution is actually numerically small [11]. I will neglect $\bar{C}_{1,2}$ in what follows.

The scalings in Eqs. (2.5) - 2.7) are based on NDA. For the $\bar{\theta}$ term, one can do better by exploiting a relation between matrix element of isospin breaking operators and $\not P T$ operators [12],

$$
\frac{\bar{g}_{0}}{F_{\pi}}=\frac{\delta m_{N}}{F_{\pi}} \frac{1-\varepsilon^{2}}{2 \varepsilon} \bar{\theta}=1.5 \cdot 10^{-2} \bar{\theta},
$$


where $\delta m_{N}$ is the strong interaction contribution to the neutron-proton mass difference, for which we used the value in Ref. [13]. For couplings in the subleading EFT Lagrangian, e.g. $\bar{g}_{1}$ or $\bar{d}_{1,0}$, the relations between $P T$ and $P P T$ operators is still formally valid, but in practice not useful [10]. A recent estimate [14] found $\bar{g}_{1}$ to be less suppressed than expected by NDA, $\bar{g}_{1} / \bar{g}_{0} \sim 0.1$, roughly a factor of ten larger than expected. For the low-energy constants (LEC) $\bar{d}_{0,1}$, I will just use NDA. Also, I will not go beyond NDA for all the PPT couplings stemming from dimension-six sources.

\section{Nuclear Observables}

The EFT Lagrangian (2.4) can be used to compute $\not P T$ nuclear observables. I focus here on the EDMs of the nucleon, the deuteron, and of ${ }^{3} \mathrm{He}$ and ${ }^{3} \mathrm{H}$.

In the one-nucleon sector, the electric dipole form factor (EDFF) can be systematically computed in an expansion in powers of $Q / M_{\mathrm{QCD}}$. The EDFF receives contributions at tree level from the short-range operators $\bar{d}_{0,1}$, and at one loop from the $P P T$ pion-nucleon couplings $\bar{g}_{0,1}$. The additional loop costs two powers of $Q / M_{\mathrm{QCD}}$, and it can be neglected if $\bar{g}_{0,1}$ are of the same order as, or smaller than, the LECs $\bar{d}_{0,1}$. For the $\chi$ ISs and the qEDM, therefore, the nucleon EDFF is at leading order (LO) completely determined by two LECs

$$
d_{0}=\bar{d}_{0}, \quad d_{1}=\bar{d}_{1},
$$

and is momentum independent. The first contribution to the momentum dependence only appears at NNLO, and it is governed by the scale $M_{\mathrm{QCD}}$. With the NDA estimates in Eq. 2.7), the current bound on the neutron EDM can be converted in bounds on the new physics scale: $\delta / M_{\mp}^{2} \lesssim\left(10^{2} \mathrm{TeV}\right)^{-2}$ for the $\mathrm{qEDM}$, and $w / M_{T}^{2} \lesssim\left(10^{3} \mathrm{TeV}\right)^{-2}$ for $\chi \mathrm{ISs}$.

For the $\bar{\theta}$ term, the qCEDM and the FQLR, the $M_{\mathrm{QCD}}^{2}$ suppression causes the loop diagrams and the contribution from $\bar{d}_{0,1}$ to appear at the same order. For these sources, at NLO [15, 16, 17]

$$
\begin{aligned}
& d_{0}=\bar{d}_{0}+\frac{e g_{A} \bar{g}_{0}}{\left(2 \pi F_{\pi}\right)^{2}} \frac{3 \pi}{4} \frac{m_{\pi}}{m_{N}}\left(1+\frac{\bar{g}_{1}}{3 \bar{g}_{0}}\right), \\
& d_{1}=\bar{d}_{1}+\frac{e g_{A} \bar{g}_{0}}{\left(2 \pi F_{\pi}\right)^{2}}\left[L-\log \frac{m_{\pi}^{2}}{\mu^{2}}+\frac{5 \pi}{4} \frac{m_{\pi}}{m_{N}}\left(1+\frac{\bar{g}_{1}}{5 \bar{g}_{0}}\right)\right] .
\end{aligned}
$$

At LO, the isoscalar EDFF is momentum independent, and fixed by $\bar{d}_{0}$. The first non-analytic contributions arise at NLO, where one also finds the first contribution to the momentum dependence.

Pion loops contribute to the isovector EDFF already at LO, where the only relevant pionnucleon coupling is $\bar{g}_{0}$. The diagrams are logarithmically divergent, with the divergence encoded by $L$. Renormalization requires the counterterm $\bar{d}_{1}$ to be of the same order as the loop, in agreement with power counting. The momentum dependence of the EDFF comes entirely from the loop, it is governed by $m_{\pi}^{2}$, and only depends on $\bar{g}_{0}$. At NLO, one finds the first contribution from $\bar{g}_{1}$. However, $\bar{g}_{1}$ contributes only to the EDM, not to the EDFF, and cannot easily be disentagled from the LECs $\bar{d}_{0,1}$. Since the main difference between the $\bar{\theta}$ term and the isospin-breaking qCEDM and FQLR is the relative size of $\bar{g}_{0}$ and $\bar{g}_{1}$, the nucleon EDFF alone, without further input from the lattice, does not allow to disentagle the $\bar{\theta}$ term from dimension-six chiral breaking operators.

To estimate the nucleon EDM, I need to make some assumptions. Though they contribute at the same order, I do not expect cancellations between the short-range contributions, analytic in 
$m_{\pi}^{2}$, and the long-range, non-analytic in $m_{\pi}^{2}$. I estimate $d_{1}$ by neglecting the LEC $\bar{d}_{1}$, and setting $\mu \sim m_{N}$, to obtain $d_{1} \sim 0.15 \bar{g}_{0} / F_{\pi} e \mathrm{fm}$. For $d_{0}$, the non-analytic piece is subleading, and provides a lower bound $\left|d_{0}\right| \gtrsim 0.01 \bar{g}_{0} / F_{\pi} e \mathrm{fm}$, roughly a factor of 3 smaller than the NDA estimate for $\bar{d}_{0}$. For to the $\bar{\theta}$ term, using Eq. (2.8) the bound on the neutron EDM can be converted on a limit on $\bar{\theta}$, $\bar{\theta}<10^{-10}$. For the qCEDM and FQLR, the bound on the neutron EDM, combined with the NDA estimate for $\bar{g}_{0} / F_{\pi}$, indicates a new physics scale of several TeV, e.g. $\tilde{\delta} / M_{T}^{2} \lesssim\left(10^{2} \mathrm{TeV}\right)^{-2}$.

In summary, for all the $\not P T$ operators in Eqs. (2.1) and (2.2), the nucleon EDM depends on at least two undetermined LECs. A measurement of the EDM of proton and neutron in the next generation of experiments, while clearly a signal of $P$ T beyond the phase of CKM, can be fitted by any of the sources I consider. In particular it would not allow to determine whether the culprit for $C P$ violation is the QCD $\bar{\theta}$ term, of physics beyond the SM.

To this goal, more low-energy observables are needed. A promising direction is to investigate the EDMs of light nuclei. The EDM of a bound state receives several contributions: from the EDMs of the constituents, $P$ T corrections to the bound state wavefunction, two- or many-body $P P T$ currents, etc. The power counting of the EFT allows to systematically organize these contributions. For systems with $A \geq 2$, the infrared enhancement of nucleon lines with almost on-shell nucleons has, as a main consequence, the result that adding extra loops, with pion exchanges between different nucleon lines, only costs powers of $Q / M_{\mathrm{NN}} \sim Q / F_{\pi}$, rather than $Q / M_{\mathrm{QCD}}$. Therefore, we expect that in the case of the $\bar{\theta}$ term, the qCEDM and the FQLR, the EDMs of light nuclei are dominated by pion-exchange corrections to the bound state wavefunction. For $\chi$ ISs, $P P T$ corrections to the wavefunction, both from one-pion-exchange and short-range $P T$ potential, and the one-body contribution from the nucleon EDM should contribute at the same level, while for the qEDM the EDMs of light nuclei should not be significantly different from the EDMs of their constituents. These expectations can be tested on the deuteron, helion and triton.

In the case of the deuteron, because of spin and isospin selection rules, $\bar{g}_{0}$ does not contribute to the EDM at LO, while $\bar{g}_{1}$ does. The deuteron EDM $d_{d}$ is then a crucial observable to identify isospin breaking sources, e.g the qCEDM. $d_{d}$ was recently computed in chiral EFT in Refs. [18, 11, 14]. In the perturbative pion approach, Ref. [18] finds

$$
d_{d}=d_{n}+d_{p}-e \frac{\bar{g}_{1}}{m_{\pi}} \frac{m_{N}}{6 \pi F_{\pi}^{2}} \frac{1+\xi}{(1+2 \xi)^{2}}=2 d_{0}-0.23 \frac{\bar{g}_{1}}{F_{\pi}} e \mathrm{fm}
$$

where $d_{n}+d_{p}=2 d_{0}$, and $\xi=\gamma / m_{\pi}$, with $\gamma$ the deuteron binding momentum, $\gamma=45 \mathrm{MeV}$. The pion-exchange contribution dominates for the qCEDM and the FQLR, with an expected enhancement of a factor $M_{\mathrm{QCD}}^{2} / m_{\pi} M_{\mathrm{NN}} \sim 10$ with respect to $d_{0}$. To be more quantitative, one needs to know the precise dependence of $\bar{d}_{0}$ and $\bar{g}_{1}$ on the couplings $\tilde{\delta}$ and $\xi$. In absence of that, NDA suggests $\bar{d}_{0} \sim 0.03 \bar{g}_{1} / F_{\pi} e \mathrm{fm}$, so that $d_{0}$ should be no more than $30 \%$ of the pion-exchange contribution.

In the case of the $\bar{\theta}$ term and the $\chi$ ISs the pion-exchange contribution is suppressed, by a factor $Q / M_{\mathrm{NN}} \sim 1 / 3$, with respect to the one-body piece. Resorting again to NDA the contribution of $\bar{g}_{1}$ should be about $10 \%$ of $d_{0}$. Obviously, the LEC $\bar{d}_{0}$ could be smaller than NDA, and then the two components of Eq. (3.4) could be of the same size. In this case, however, one should also observe a big difference between $d_{0}$ and $d_{1}$. For the qEDM $\bar{g}_{1}$ is small, and $d_{d}$ should not differ from $2 d_{0}$.

From this discussion, we see that only for the qCEDM and FQLR $d_{d}$ is expected to differ significantly from $2 d_{0}$. The observation of the nucleon and deuteron EDM, then, would give important 
hints to identify, or exclude, these two sources as the dominant mechanism of $\not P T$.

With more nuclei, one can collect more pieces of the puzzle. ${ }^{3} \mathrm{He}$ and ${ }^{3} \mathrm{H}$ are interesting because there is no selection rule suppressing the contribution of $\bar{g}_{0}$. A calculation that used an "hybrid" approch, i.e the EFT PPT potential of Ref. [19], and high-precision PT phenomenological potentials for the three-nucleon wavefunction, was performed in Refs. [20, 11], and found

$$
\frac{d_{3} \mathrm{H}+d_{3} \mathrm{He}}{2}=0.84 d_{0}-0.28 \frac{\bar{g}_{1}}{F_{\pi}} e \mathrm{fm}, \quad \frac{d_{3 \mathrm{H}}-d_{3} \mathrm{He}}{2}=0.94 d_{1}+0.15 \frac{\bar{g}_{0}}{F_{\pi}} e \mathrm{fm},
$$

where I am neglecting the numerically small contributions of $\bar{C}_{1,2}$, and showing results for AV18. The discussion for the isoscalar combination, $d_{3_{\mathrm{H}}}+d_{3_{\mathrm{He}}}$, parallels very closely the one for the deuteron. For the qCEDM and FQLR, $d_{0}$ provides a $10 \%$ correction to the contribution of $\bar{g}_{1}$, while for all the other sources no large deviation from $d_{0}$ is expected.

For the isovector combination $d_{3 \mathrm{H}}-d_{3 \mathrm{He}}$, the enhancement of $d_{1}$ due to the chiral $\log$, and the smallness of the nuclear matrix element in front of $\bar{g}_{0}$, cause the one- and two-body contributions to be of similar size for the $\bar{\theta}$ term, the qCEDM, and the FQLR. Contrary to the power counting expectation, we cannot therefore neglect $d_{1}$. For the $\chi$ ISs, even though one expects $\bar{g}_{0,1}$ to be important, the smallness of their nuclear matrix elements causes the EDM to be dominated by $d_{1}$. For the qEDM, as usual, no deviation from $d_{1}$ is expected. $d_{3} \mathrm{H}-d_{3} \mathrm{He}$ would then help to understand if the dominant $\not P T$ mechanism generates a large $\bar{g}_{0}$, and to isolate the $\bar{\theta}$ term.

\section{Conclusion}

The observation of an EDM in the next generation of experiments would put to an end a hunt began with Purcell and Ramsey's experiment more than seventy years ago. At the same time, it would open the question on the fundamental mechanism behind the observed $\not P T$. We have discussed how chiral and isospin properties of $\not P T$ operator at the quark-gluon level imply qualitatively different relations between the nucleon, deuteron and three-nucleon EDMs. If experimentally observed, these relations would provide important clues on the nature of the dominant $\not P T$ mechanism(s).

\section{Acknowledgments}

I would like to thank J. de Vries, U. van Kolck, R. Timmermans, C. Maekawa, C.-P. Liu, I. Stetcu, R. Higa, and W. Hockings for the collaborations on various projects presented here. This research was supported by the US DOE under contract DE-AC02-05CH11231.

\section{References}

[1] M. Pospelov and A. Ritz, "Electric dipole moments as probes of new physics," Annals Phys. 318 (2005) 119 [hep-ph/0504231].

[2] C. A. Baker, D. D. Doyle, P. Geltenbort, K. Green, M. G. D. van der Grinten, P. G. Harris, P. Iaydjiev and S. N. Ivanov et al., "An Improved experimental limit on the electric dipole moment of the neutron,” Phys. Rev. Lett. 97 (2006) 131801 [hep-ex/0602020]. 
[3] Y. F. Orlov, W. M. Morse and Y. K. Semertzidis, "Resonance method of electric-dipole-moment measurements in storage rings,” Phys. Rev. Lett. 96 (2006) 214802 [hep-ex/0605022].

[4] V. Baluni, “CP Violating Effects in QCD,” Phys. Rev. D 19 (1979) 2227.

[5] B. Grzadkowski, M. Iskrzynski, M. Misiak and J. Rosiek, "Dimension-Six Terms in the Standard Model Lagrangian,” JHEP 1010 (2010) 085 [arXiv:1008.4884 [hep-ph]].

[6] J. de Vries, E. Mereghetti, R. G. E. Timmermans and U. van Kolck, "The Effective Chiral Lagrangian From Dimension-Six Parity and Time-Reversal Violation,” arXiv:1212.0990 [hep-ph].

[7] J. Ng and S. Tulin, “D versus d: CP Violation in Beta Decay and Electric Dipole Moments,” Phys. Rev. D 85 (2012) 033001 [arXiv:1111.0649 [hep-ph]].

[8] F. Berruto, T. Blum, K. Orginos and A. Soni, "Calculation of the neutron electric dipole moment with two dynamical flavors of domain wall fermions," Phys. Rev. D 73 (2006) 054509 [hep-lat/0512004].

[9] E. Shintani, S. Aoki, N. Ishizuka, K. Kanaya, Y. Kikukawa, Y. Kuramashi, M. Okawa and Y. Tanigchi et al., "Neutron electric dipole moment from lattice QCD,” Phys. Rev. D 72 (2005) 014504 [hep-lat/0505022].

[10] E. Mereghetti, W. H. Hockings and U. van Kolck, "The Effective Chiral Lagrangian From the Theta Term,” Annals Phys. 325 (2010) 2363 [arXiv:1002.2391 [hep-ph]].

[11] J. de Vries, R. Higa, C. -P. Liu, E. Mereghetti, I. Stetcu, R. G. E. Timmermans and U. van Kolck, “Electric Dipole Moments of Light Nuclei From Chiral Effective Field Theory,” Phys. Rev. C 84, 065501 (2011) [arXiv:1109.3604 [hep-ph]].

[12] R. J. Crewther, P. Di Vecchia, G. Veneziano and E. Witten, "Chiral Estimate of the Electric Dipole Moment of the Neutron in Quantum Chromodynamics,” Phys. Lett. B 88 (1979) 123 [Erratum-ibid. B 91 (1980) 487].

[13] S. R. Beane, K. Orginos and M. J. Savage, "Strong-isospin violation in the neutron-proton mass difference from fully-dynamical lattice QCD and PQQCD,” Nucl. Phys. B 768 (2007) 38 [hep-lat/0605014].

[14] J. Bsaisou, C. Hanhart, S. Liebig, U. -G. Meissner, A. Nogga and A. Wirzba, "The electric dipole moment of the deuteron from the QCD $\theta$-term,” arXiv:1209.6306 [hep-ph].

[15] W. H. Hockings and U. van Kolck, “The Electric dipole form factor of the nucleon,” Phys. Lett. B 605 (2005) 273 [nucl-th/0508012].

[16] K. Ottnad, B. Kubis, U. -G. Meissner and F. -K. Guo, "New insights into the neutron electric dipole moment,” Phys. Lett. B 687 (2010) 42 [arXiv:0911.3981 [hep-ph]].

[17] E. Mereghetti, J. de Vries, W. H. Hockings, C. M. Maekawa and U. van Kolck, "The Electric Dipole Form Factor of the Nucleon in Chiral Perturbation Theory to Sub-leading Order,” Phys. Lett. B 696 (2011) 97 [arXiv:1010.4078 [hep-ph]].

[18] J. de Vries, E. Mereghetti, R. G. E. Timmermans and U. van Kolck, "Parity- and Time-Reversal-Violating Form Factors of the Deuteron,” Phys. Rev. Lett. 107, 091804 (2011) [arXiv:1102.4068 [hep-ph]].

[19] C. M. Maekawa, E. Mereghetti, J. de Vries and U. van Kolck, "The Time-Reversal- and Parity-Violating Nuclear Potential in Chiral Effective Theory,” Nucl. Phys. A 872 (2011) 117 [arXiv:1106.6119 [nucl-th]].

[20] I. Stetcu, C. -P. Liu, J. L. Friar, A. C. Hayes and P. Navratil, "Nuclear Electric Dipole Moment of He-3,” Phys. Lett. B 665 (2008) 168 [arXiv:0804.3815 [nucl-th]]. 